Louisiana State University

LSU Digital Commons

Faculty Publications

Department of Physics \& Astronomy

3-20-2016

\title{
STELLAR ACTIVITY and EXCLUSION of the OUTER PLANET in the HD 99492 SYSTEM
}

Stephen R. Kane

San Francisco State University

Badrinath Thirumalachari

San Francisco State University

Gregory W. Henry

Tennessee State University

Natalie R. Hinkel

San Francisco State University

Eric L.N. Jensen

Swarthmore College

See next page for additional authors

Follow this and additional works at: https://digitalcommons.Isu.edu/physics_astronomy_pubs

\section{Recommended Citation}

Kane, S., Thirumalachari, B., Henry, G., Hinkel, N., Jensen, E., Boyajian, T., Fischer, D., Howard, A., Isaacson, H., \& Wright, J. (2016). STELLAR ACTIVITY and EXCLUSION of the OUTER PLANET in the HD 99492 SYSTEM. Astrophysical Journal Letters, 820 (1) https://doi.org/10.3847/2041-8205/820/1/L5

This Article is brought to you for free and open access by the Department of Physics \& Astronomy at LSU Digital Commons. It has been accepted for inclusion in Faculty Publications by an authorized administrator of LSU Digital Commons. For more information, please contact ir@lsu.edu. 


\section{Authors}

Stephen R. Kane, Badrinath Thirumalachari, Gregory W. Henry, Natalie R. Hinkel, Eric L.N. Jensen, Tabetha S. Boyajian, Debra A. Fischer, Andrew W. Howard, Howard T. Isaacson, and Jason T. Wright 
Swarthmore College

Works

3-14-2016

\title{
Stellar Activity And Exclusion Of The Outer Planet In The HD 99492 System
}

\author{
S. R. Kane \\ B. Thirumalachari \\ G. W. Henry \\ N. R. Hinkel \\ Eric L. N. Jensen \\ Swarthmore College, ejensen1@swarthmore.edu
}

See next page for additional authors

Follow this and additional works at: https://works.swarthmore.edu/fac-physics

Part of the Astrophysics and Astronomy Commons

Let us know how access to these works benefits you

\section{Recommended Citation}

S. R. Kane, B. Thirumalachari, G. W. Henry, N. R. Hinkel, Eric L. N. Jensen, T. S. Boyajian, D. A. Fischer, A. W. Howard, H. T. Isaacson, and J. T. Wright. (2016). "Stellar Activity And Exclusion Of The Outer Planet In The HD 99492 System". Astrophysical Journal Letters. Volume 820, Issue 1. DOI: 10.3847/2041-8205/820/1/ L5

https://works.swarthmore.edu/fac-physics/246

This work is brought to you for free by Swarthmore College Libraries' Works. It has been accepted for inclusion in Physics \& Astronomy Faculty Works by an authorized administrator of Works. For more information, please contact myworks@swarthmore.edu. 


\section{Authors}

S. R. Kane, B. Thirumalachari, G. W. Henry, N. R. Hinkel, Eric L. N. Jensen, T. S. Boyajian, D. A. Fischer, A. W. Howard, H. T. Isaacson, and J. T. Wright 


\section{STELLAR ACTIVITY AND EXCLUSION OF THE OUTER PLANET IN THE HD 99492 SYSTEM}

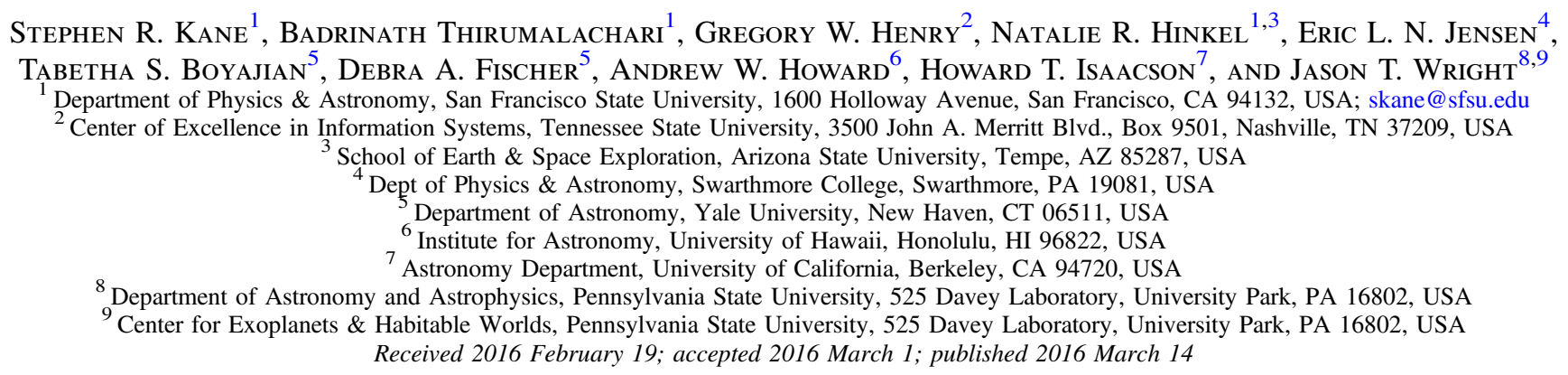

\section{ABSTRACT}

A historical problem for indirect exoplanet detection has been contending with the intrinsic variability of the host star. If the variability is periodic, it can easily mimic various exoplanet signatures, such as radial velocity (RV) variations that originate with the stellar surface rather than the presence of a planet. Here we present an update for the HD 99492 planetary system, using new RV and photometric measurements from the Transit Ephemeris Refinement and Monitoring Survey. Our extended time series and subsequent analyses of the Ca II H\&K emission lines show that the host star has an activity cycle of $\sim 13$ years. The activity cycle correlates with the purported orbital period of the outer planet, the signature of which is thus likely due to the host star activity. We further include a revised Keplerian orbital solution for the remaining planet, along with a new transit ephemeris. Our transit-search observations were inconclusive.

Key words: planetary systems - stars: individual (HD 99492) - techniques: photometric - techniques: radial velocities

\section{INTRODUCTION}

The radial velocity (RV) technique remains one of the most successful methods for the discovery of exoplanetary systems. At the present time, more than 500 exoplanets have been discovered using the RV technique, including a vast range of multi-planet systems and orbital configurations. The success of this method is greatly dependent upon the ability to accurately characterize the properties of the host star. In particular, the evolution of star spots, magnetic fields, and pulsations have well-studied effects on stellar RV variations (Saar \& Donahue 1997; Queloz et al. 2001; Desort et al. 2007; Hébrard et al. 2014). There have been numerous recent cases where stellar activity has posed a significant problem in correctly interpreting RV data (Hatzes 2013; Robertson et al. 2014, 2015; Hatzes et al. 2015).

One source of activity-induced RV variations is due to stellar activity cycles, analogous to the 11 year solar cycle. Dravins (1985) predicted such a correlation, and Deming et al. (1987) reported the detection of such a correlation in the solar CO lines at $2.3 \mu \mathrm{m}$, and inferred an amplitude of $30 \mathrm{~m} \mathrm{~s}^{-1}$ from the effect. Wright et al. (2008) argued that experience with the hundreds of Sun-like stars from the California Planet Survey (CPS) showed that such effects are not so strong, and that activity cycles were probably not to blame for a $\sim 15 \mathrm{~m} \mathrm{~s}^{-1} \mathrm{RV}$ variation in phase with an activity cycle in HD 154345. Similar high-amplitude RV activity correlations in individual targets have been reported by Moutou et al. (2011), Carolo et al. (2014), and Robertson et al. (2013). Nonetheless, for most stars such correlations are small or absent, as argued by Wright et al. (2008) and Santos et al. (2010).
The star HD 99492 is an early-K dwarf in a binary orbit with HD 99491 (also known as 83 Leonis B and A, respectively). HD 99492 has a parallax of $55.7 \pm 1.46$ marcsec and a distance of $17.96 \pm 0.47 \mathrm{pc}$ (van Leeuwen 2007a, 2007b). The mean angular separation of the stellar components is 40 " 76 , leading to an average projected separation of $\sim 730$ au. HD 99492 was found to harbor a $0.1 M_{J}$ planet in a 17 day orbit by Marcy et al. (2005). The best-fit Keplerian orbital solution at that time included a linear trend to account for a possible second companion in the system. The orbital elements were updated by Meschiari et al. (2011), who claimed to have resolved the separate orbit of an outer planet with a period of $\sim 5000$ days.

Here we present new results for the system that reveal an activity cycle in the star and further show that stellar activity amply explains the signature of the outer planet (c). Section 2 provides new fundamental stellar parameters, including spectral analysis, discussion of element abundances, and activity indices from the complete data set of $130 \mathrm{Keck} /$ HIRES spectra. Section 3 presents our revised Keplerian orbital solution, including the correlation of the outer planet signature with the activity indices. Section 4 includes photometry from five observing seasons acquired over a span of 11 years. The photometric data confirms the absence of brightness variations in phase with the orbital period of planet $b$, thus confirming that the RV variations in HD 99492 on a 17 day cycle are due to planetary reflex motion. Our limited number of brightness measurements near the predicted phase of planetary transit show no evidence for a transit but fall short of ruling them out. We provide concluding remarks in Section 5. 
Table 1

System Parameters

\begin{tabular}{|c|c|}
\hline Parameter & Value \\
\hline \multicolumn{2}{|l|}{ HD 99492} \\
\hline$V$ & 7.58 \\
\hline$B-V$ & 1.0 \\
\hline Distance (pc) & $55.7 \pm 1.46$ \\
\hline$T_{\text {eff }}(\mathrm{K})$ & $4929 \pm 44$ \\
\hline $\log g$ & $4.57 \pm 0.06$ \\
\hline$v \sin i\left(\mathrm{~km} \mathrm{~s}^{-1}\right)$ & $0.41 \pm 0.5$ \\
\hline$[\mathrm{Fe} / \mathrm{H}](\mathrm{dex})$ & $0.3 \pm 0.03$ \\
\hline$M_{\star}\left(M_{\odot}\right)$ & $0.85 \pm 0.02$ \\
\hline$R_{\star}\left(R_{\odot}\right)$ & $0.78 \pm 0.02$ \\
\hline Age (Gyr) & $4.8 \pm 4.1$ \\
\hline \multicolumn{2}{|l|}{ HD 99492 b } \\
\hline$P$ (days) & $17.054 \pm 0.003$ \\
\hline$T_{c}^{\mathrm{a}}(\mathrm{JD}-2,440,000)$ & $17367.776 \pm 0.855$ \\
\hline$T_{p}^{\mathrm{b}}(\mathrm{JD}-2,440,000)$ & $13776.317 \pm 3.392$ \\
\hline$e$ & $0.07 \pm 0.06$ \\
\hline$\omega(\operatorname{deg})$ & $240.7 \pm 75.4$ \\
\hline$K\left(\mathrm{~m} \mathrm{~s}^{-1}\right)$ & $6.98 \pm 0.53$ \\
\hline$M_{p} \sin i\left(M_{J}\right)$ & $0.079 \pm 0.006$ \\
\hline$a(\mathrm{au})$ & $0.123 \pm 0.001$ \\
\hline \multicolumn{2}{|l|}{ System Properties } \\
\hline$\gamma\left(\mathrm{m} \mathrm{s}^{-1}\right)$ & $-1.49 \pm 0.37$ \\
\hline \multicolumn{2}{|c|}{ Measurements and Model } \\
\hline$N_{\text {obs }}$ & 130 \\
\hline $\mathrm{rms}\left(\mathrm{m} \mathrm{s}^{-1}\right)$ & 4.33 \\
\hline$\chi_{\text {red }}^{2}$ & 1.03 \\
\hline
\end{tabular}

Notes.

Time of mid-transit.

b Time of periastron passage.

\section{STELLAR PROPERTIES}

\subsection{Fundamental Parameters}

The fundamental properties of HD 99492 have been previously determined, for example, by Valenti \& Fischer (2005) and Takeda et al. (2007). We used an upgraded version of the Spectroscopy Made Easy (SME) package to model a Keck/HIRES spectrum of HD 99492. Details of the SME package may be found in Valenti \& Piskunov (1996) and Valenti \& Fischer (2005). Briefly, SME uses an iterative technique that combines a model atmosphere analysis with Yonsei-Yale model isochrones (Demarque et al. 2004) that utilize Hipparcos photometry and distances (van Leeuwen 2007a, 2007b). This approach produces a selfconsistent convergence with the measured surface gravity (Valenti et al. 2009).

The results of our analysis are shown in Table 1, including values for the surface gravity $\log g$, rotational velocity $v \sin i$, atmospheric abundance $[\mathrm{Fe} / \mathrm{H}]$, effective temperature $T_{\text {eff }}$, and stellar isochrone solution (mass $M_{\star}$, radius $R_{\star}$, and age). These parameters are consistent with previous estimates of the stellar properties and demonstrate that HD 99492 is a late-G/early-K dwarf with an age similar to the Sun.

\subsection{Stellar Abundances}

The element abundances of HD 99492 have been measured by only two groups to date, namely Valenti \& Fischer (2005) and Petigura \& Marcy (2011). To correct for varying solar abundance normalizations, per the analysis within the Hypatia Catalog (Hinkel et al. 2014), each data set was re-normalized to the Lodders et al. (2009) scale. The $[\mathrm{Fe} / \mathrm{H}]$ measurement of both groups is $0.40 \mathrm{dex}$, since Petigura \& Marcy (2011) adopted the stellar parameters and iron abundance from Valenti \& Fischer (2005) in their analysis. From Petigura \& Marcy (2011), $[\mathrm{O} / \mathrm{H}]=0.25$ dex while Valenti \& Fischer (2005) determined $[\mathrm{Na} / \mathrm{H}]=0.41,[\mathrm{Si} / \mathrm{H}]=0.34 \mathrm{dex}$, $[\mathrm{Ti} / \mathrm{H}]=0.28 \mathrm{dex}$, and $[\mathrm{Ni} / \mathrm{H}]=0.38 \mathrm{dex}$. These results reveal a star that is markedly super-solar in both the volatile and refractory elements.

\subsection{Stellar Activity}

HD 99492 has been spectroscopically monitored using the HIRES echelle spectrometer (Vogt et al. 1994) on the $10.0 \mathrm{~m}$ Keck I telescope as part of the CPS. For Keck/HIRES instrument configuration details, see Wright et al. (2004) and Howard et al. (2009). Our complete HIRES data set contains 130 measurements spanning over 18 years, extending the time baseline of the data reported by Meschiari et al. (2011) by over 5 years. The pipeline that extracts the RVs from the spectra (see Section 3) also extracts $\mathrm{Ca}$ II $\mathrm{H} \& \mathrm{~K}$ line-profile variations and provides an index of stellar activity (Noyes et al. 1984). These data are calibrated to the Mt. Wilson S-values, defined as the ratio of the sum of the flux in the $H \& K$ line cores to the sum of the two continuum bands on either side (Wilson 1968). We include data acquired both before and after the upgrade of the HIRES CCD in 2004 August (Isaacson \& Fischer 2010), taking into account the offset between pre-2004 and post-2004 calibrated data sets.

The time series of S-values are shown in the left panel of Figure 1. The periodic variation in $\mathrm{S}$ indicates that we have observed just over one complete cycle of stellar activity in the host star. To quantify the variation, we performed a Fourier analysis of the time series, resulting in the periodogram shown in the right panel of Figure 1. This analysis reveals a broad peak in the power spectrum that lies between 3000 and 5000 days, with maximum power occurring at $\sim 3650$ days. The S-value periodicity is thus consistent with the HD 99492c orbital period of $4970 \pm 744$ days determined by Meschiari et al. (2011). We elaborate further on the correlation between stellar activity and possible planetary signature in Section 3.

\section{AN UPDATE TO THE PLANETARY SYSTEM}

The RV measurements were extracted from the Keck/ HIRES data with the use of an iodine cell mounted at the spectrometer entrance slit as a robust source of wavelength calibration (Marcy \& Butler 1992; Valenti et al. 1995). The modeling procedure for the Doppler shift of each stellar spectrum with respect to the iodine spectrum is described further in Howard et al. (2009). The discovery orbital solution for the HD 99492 system by Marcy et al. (2005) included a linear trend component. The $93 \mathrm{RV}$ measurements utilized by Meschiari et al. (2011) used a two-planet orbital solution to account for the previously noted linear trend. A two-planet fit to our expanded data set is able to recover an orbital solution similar to that previously found by Meschiari et al. (2011). 

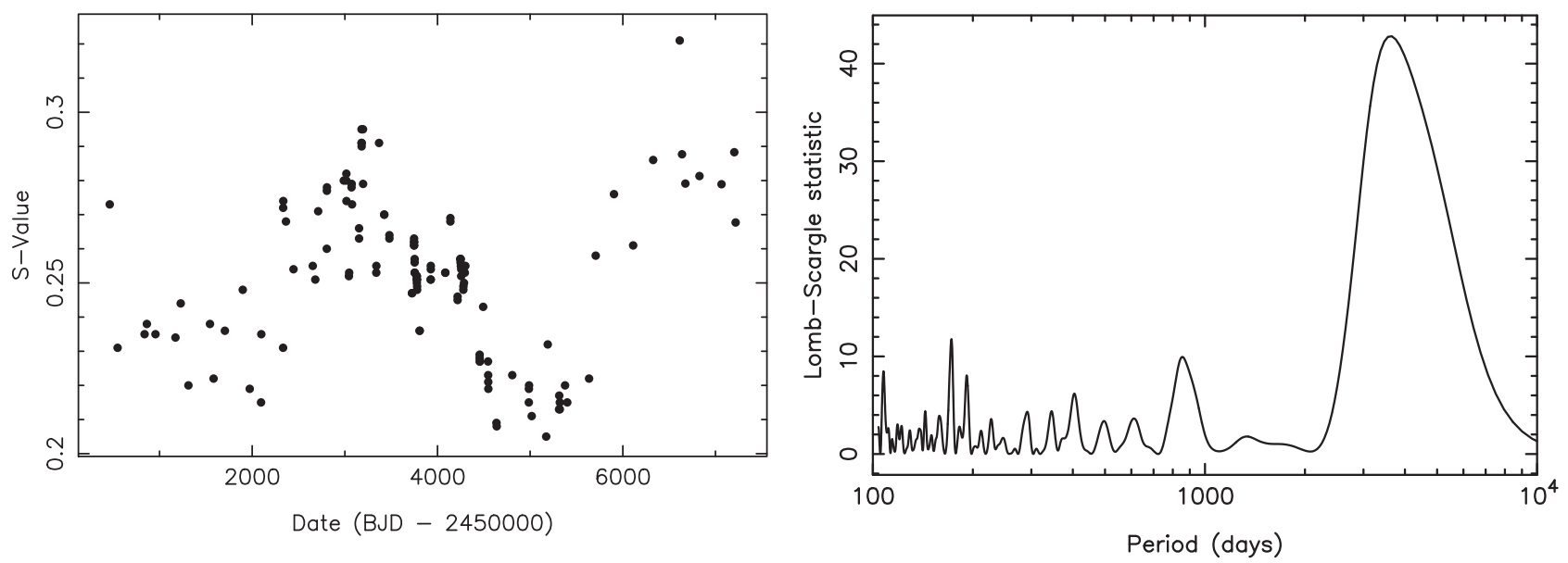

Figure 1. Left: HD 99492 S-values determined from the complete time series of Keck/HIRES spectra. Right: the periodogram resulting from a Fourier analysis of the HD 99492 S-values, revealing a broad peak between 3000 and 5000 days.

Considering the periodic stellar activity described in Section 2.3 as a source for the previously observed linear trend and purported second planet, we performed a single-planet fit to our data set of $130 \mathrm{RV}$ measurements, both with and without a linear trend included. These fits were carried out using RVLIN, a partially linearized, least-squares fitting procedure described in Wright \& Howard (2009). The uncertainties in the resulting orbital parameters were estimated using the BOOTTRAN bootstrapping routines described in Wang et al. (2012). We included a stellar jitter noise component of $4 \mathrm{~m} \mathrm{~s}^{-1}$ in quadrature with the measurement uncertainties (Wright 2005; Butler et al. 2006). With our new data set and its increased timespan, we find no evidence of a significant difference between the orbital fits that do and do not include a linear trend. We thus adopt the solution without the linear trend for which the complete orbital solution is shown in Table 1 and in the top panel of Figure 2. Note that the $\gamma$ parameter shown in Table 1 is the systemic velocity of the system with respect to the zero point of the extracted RVs and thus is the systemic velocity relative to the template spectrum. The complete RV data set of 130 measurements of HD 99492 is listed in Table 2.

To investigate further the impact of stellar activity on a twoplanet solution (see Section 2.3), we compared the S-values with the RV residuals of the single-planet solution shown in Table 1. The resulting correlation diagram is shown in the bottom panel of Figure 2. We quantified the significance of the correlation using the Spearman rank correlation coefficient. The Spearman coefficient lies in the range $-1<r_{s}<1$, and, in turn, gives the probability that the two quantities being examined are not correlated. The Spearman coefficient for the data shown in the bottom panel of Figure 2 is $r_{s}=0.39$, indicative of a positive correlation. The corresponding probability that the residuals of the single-planet solution and the $\mathrm{S}$-values would produce the observed correlation if those quantities were in fact uncorrelated is $1.2 \times 10^{-5}$. We conducted a further test via an extensive Monte-Carlo simulation that performs a Fisher-Yates shuffle, randomizing the order of the residual data values. For each realization, the Spearman's rank correlation coefficient and probability were recalculated. This test resulted in a 0.5 probability of null correlation, indicating that the correlation found above is robust. This implies, in turn, that the second planet claimed by Meschiari et al. (2011) is instead the result of stellar activity.
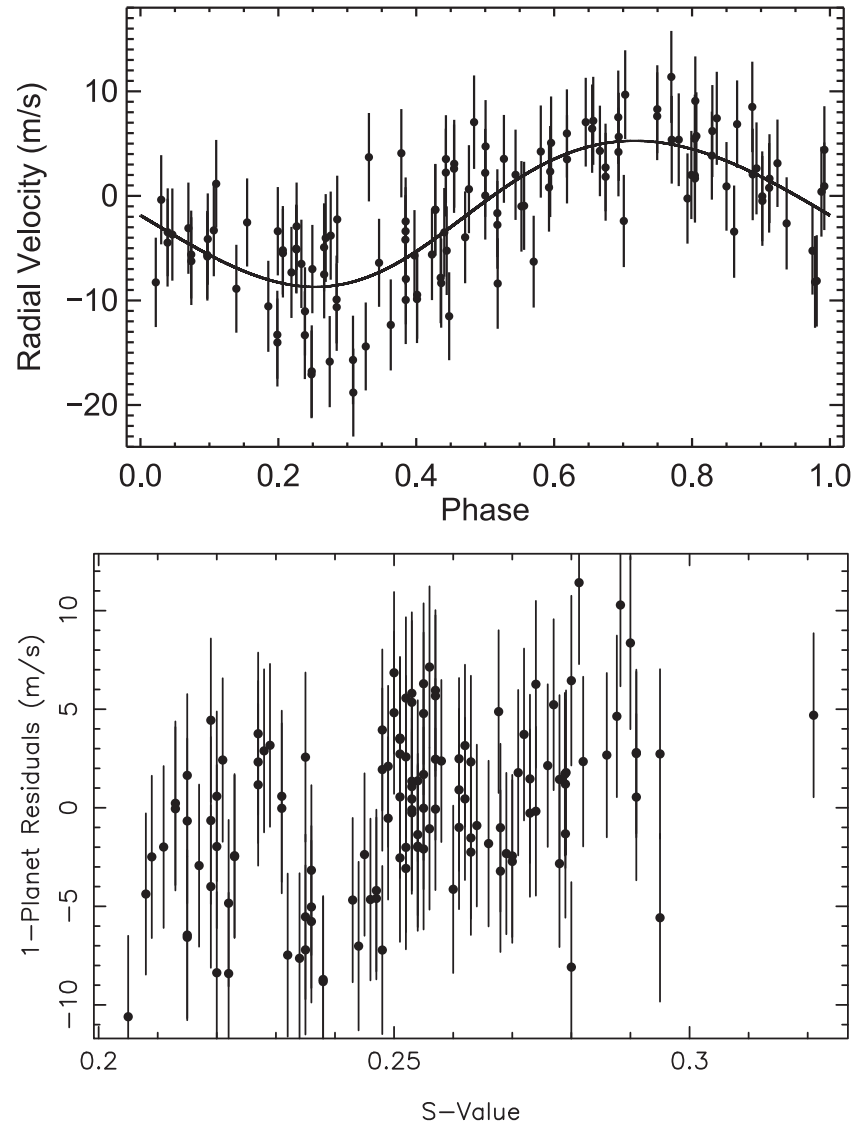

Figure 2. Top: the complete $130 \mathrm{RV}$ measurement data set phased on the bestfit Keplerian orbital solution for a single-planet system (see Table 1). Bottom: the residuals from the best-fit solution plotted against the activity indices described in Section 2.3. Our analysis shows that the probability of no correlation between the one-planet RV residuals and the S-values is $1.2 \times 10^{-5}$

\section{PHOTOMETRIC OBSERVATIONS}

We observed HD 99492 photometrically as part of the Transit Ephemeris Refinement and Monitoring Survey (Kane et al. 2009) with the T12 $0.8 \mathrm{~m}$ Automatic Photoelectric Telescope (APT), one of several automated telescopes operated by Tennessee State University (TSU) at Fairborn Observatory in southern Arizona. The T12 APT is equipped with a 
Table 2

HD 99492 Radial Velocities

\begin{tabular}{|c|c|c|}
\hline $\begin{array}{l}\text { Date } \\
\text { (BJD-2,440,000) }\end{array}$ & $\begin{array}{c}\mathrm{RV} \\
\left(\mathrm{m} \mathrm{s}^{-1}\right)\end{array}$ & $\begin{array}{c}\sigma \\
\left(\mathrm{m} \mathrm{s}^{-1}\right)\end{array}$ \\
\hline 10462.113958 & -3.09 & 1.49 \\
\hline 10546.987859 & -3.70 & 1.62 \\
\hline 10837.932535 & -3.30 & 1.56 \\
\hline 10862.898993 & -6.29 & 1.57 \\
\hline 10955.876644 & -8.27 & 1.21 \\
\hline 11172.101597 & -2.40 & 1.62 \\
\hline 11228.035903 & -8.13 & 1.51 \\
\hline 11311.816319 & 2.63 & 1.59 \\
\hline 11544.172650 & -8.38 & 1.39 \\
\hline 11582.974942 & -0.25 & 1.35 \\
\hline 11704.805914 & -2.63 & 1.62 \\
\hline 11898.154005 & -15.85 & 1.48 \\
\hline 11973.053090 & 4.30 & 1.39 \\
\hline 12095.752049 & -3.42 & 1.63 \\
\hline 12097.753715 & -8.23 & 1.56 \\
\hline 12333.139410 & 5.37 & 1.69 \\
\hline 12334.079884 & 7.42 & 1.73 \\
\hline 12334.968322 & 2.05 & 1.53 \\
\hline 12364.068125 & 2.33 & 1.46 \\
\hline 12445.768264 & -7.95 & 1.40 \\
\hline 12654.009595 & 5.07 & 1.70 \\
\hline 12681.123484 & -10.56 & 1.46 \\
\hline 12711.858843 & 0.40 & 1.28 \\
\hline 12804.765590 & -7.83 & 1.43 \\
\hline 12805.876296 & 4.73 & 1.68 \\
\hline 12806.763634 & -1.00 & 1.39 \\
\hline 12989.171424 & -16.78 & 1.60 \\
\hline 13015.119444 & 11.38 & 1.59 \\
\hline 13016.134363 & 6.20 & 1.59 \\
\hline 13017.121921 & 8.51 & 1.38 \\
\hline 13044.127569 & -3.96 & 1.58 \\
\hline 13045.999074 & 4.24 & 1.62 \\
\hline 13071.870764 & -4.13 & 1.51 \\
\hline 13073.940752 & -7.32 & 1.47 \\
\hline 13076.983611 & -5.70 & 1.42 \\
\hline 13153.801470 & -0.04 & 1.29 \\
\hline 13153.804144 & -0.48 & 1.28 \\
\hline 13179.820787 & -1.32 & 1.46 \\
\hline 13179.824352 & -1.33 & 1.57 \\
\hline 13180.782037 & 7.05 & 1.78 \\
\hline 13181.808171 & 2.02 & 1.35 \\
\hline 13195.775914 & -12.34 & 1.46 \\
\hline 13196.794780 & -5.61 & 1.47 \\
\hline 13339.157731 & 5.37 & 0.89 \\
\hline 13340.150718 & 3.85 & 0.95 \\
\hline 13369.115093 & 3.54 & 0.92 \\
\hline 13425.000741 & 1.95 & 0.97 \\
\hline 13425.003310 & 1.67 & 1.02 \\
\hline 13480.759734 & -5.61 & 0.92 \\
\hline 13480.761887 & -6.24 & 0.90 \\
\hline 13725.101748 & -9.87 & 0.93 \\
\hline 13725.104595 & -9.46 & 0.91 \\
\hline 13747.133588 & 7.52 & 1.77 \\
\hline 13747.138218 & 4.20 & 1.10 \\
\hline 13747.145174 & 5.65 & 0.98 \\
\hline 13748.096169 & 7.62 & 0.91 \\
\hline 13748.098819 & 8.29 & 0.92 \\
\hline 13753.040035 & -4.47 & 0.91 \\
\hline 13753.043380 & -3.48 & 0.92 \\
\hline 13754.021562 & -5.64 & 0.92 \\
\hline 13754.024097 & -5.80 & 0.96 \\
\hline 13775.980868 & -3.38 & 0.96 \\
\hline 13775.983125 & -2.44 & 0.93 \\
\hline
\end{tabular}

Table 2

(Continued)

\begin{tabular}{|c|c|c|}
\hline $\begin{array}{l}\text { Date } \\
(\mathrm{BJD}-2,440,000)\end{array}$ & $\begin{array}{c}\mathrm{RV} \\
\left(\mathrm{m} \mathrm{s}^{-1}\right)\end{array}$ & $\begin{array}{c}\sigma \\
\left(\mathrm{m} \mathrm{s}^{-1}\right)\end{array}$ \\
\hline 13776.976910 & 2.23 & 0.91 \\
\hline 13776.979213 & 3.52 & 0.90 \\
\hline 13777.950347 & 2.20 & 0.95 \\
\hline 13777.952720 & 0.03 & 0.94 \\
\hline 13779.971238 & 5.97 & 0.99 \\
\hline 13779.974155 & 3.50 & 1.00 \\
\hline 13806.916794 & -13.28 & 0.95 \\
\hline 13806.918981 & -14.02 & 0.95 \\
\hline 13926.762188 & -5.05 & 0.99 \\
\hline 13926.768762 & -5.12 & 0.98 \\
\hline 13927.761840 & -9.91 & 0.90 \\
\hline 13927.764213 & -10.64 & 0.82 \\
\hline 14084.153623 & 2.60 & 1.02 \\
\hline 14084.157870 & 3.07 & 0.94 \\
\hline 14139.063102 & 2.72 & 0.86 \\
\hline 14139.064722 & 1.83 & 0.92 \\
\hline 14216.896134 & -13.32 & 0.91 \\
\hline 14216.899722 & -11.04 & 0.99 \\
\hline 14246.798900 & 0.92 & 0.85 \\
\hline 14246.800718 & 4.41 & 0.75 \\
\hline 14248.811678 & 1.16 & 0.87 \\
\hline 14250.800613 & -2.91 & 0.84 \\
\hline 14251.804815 & -2.25 & 0.82 \\
\hline 14255.765556 & -1.65 & 0.89 \\
\hline 14255.766991 & -2.77 & 0.90 \\
\hline 14277.743067 & 5.72 & 0.85 \\
\hline 14278.749942 & 6.86 & 0.83 \\
\hline 14279.748507 & 3.12 & 0.82 \\
\hline 14285.751910 & -3.80 & 0.97 \\
\hline 14294.758669 & 5.47 & 0.97 \\
\hline 14300.738970 & -2.54 & 0.96 \\
\hline 14455.109028 & -5.20 & 1.04 \\
\hline 14455.110868 & -5.48 & 1.06 \\
\hline 14456.129444 & -4.92 & 0.96 \\
\hline 14456.131400 & -7.51 & 0.92 \\
\hline 14493.134583 & -8.34 & 1.17 \\
\hline 14544.982280 & 0.64 & 0.95 \\
\hline 14546.963137 & 0.81 & 1.03 \\
\hline 14547.871944 & 7.05 & 1.10 \\
\hline 14548.847407 & 9.68 & 1.10 \\
\hline 14635.754444 & 2.00 & 0.98 \\
\hline 14638.750949 & -5.25 & 0.86 \\
\hline 14807.164861 & 0.91 & 1.08 \\
\hline 14985.837363 & -14.40 & 0.93 \\
\hline 14986.825959 & -9.95 & 1.00 \\
\hline 14987.839171 & -5.24 & 1.08 \\
\hline 15016.744103 & -8.88 & 0.93 \\
\hline 15173.123927 & -18.81 & 0.94 \\
\hline 15190.171113 & -15.69 & 1.05 \\
\hline 15311.807895 & -3.49 & 1.09 \\
\hline 15313.781452 & -0.95 & 0.97 \\
\hline 15319.842967 & 0.77 & 1.23 \\
\hline 15319.850617 & 1.65 & 1.11 \\
\hline 15376.739902 & -17.07 & 0.89 \\
\hline 15400.735697 & 6.43 & 1.01 \\
\hline 15635.955861 & -11.51 & 0.94 \\
\hline 15707.736240 & 7.18 & 0.95 \\
\hline 15905.166211 & -6.50 & 1.01 \\
\hline 16111.736847 & -6.40 & 0.95 \\
\hline 16328.051766 & -0.37 & 1.17 \\
\hline 16614.127514 & 9.08 & 1.15 \\
\hline 16639.094368 & -4.03 & 0.91 \\
\hline 16675.173277 & -4.19 & 1.18 \\
\hline
\end{tabular}


Table 2

(Continued)

\begin{tabular}{lrc}
\hline \hline $\begin{array}{l}\text { Date } \\
\text { (BJD-2,440,000) }\end{array}$ & $\begin{array}{c}\mathrm{RV} \\
\left(\mathrm{m} \mathrm{s}^{-1}\right)\end{array}$ & $\begin{array}{c}\sigma \\
\left(\mathrm{m} \mathrm{s}^{-1}\right)\end{array}$ \\
\hline 16827.757817 & 3.70 & 1.03 \\
17065.116057 & -7.00 & 1.02 \\
17203.750309 & 4.08 & 1.01 \\
17217.748571 & -3.38 & 1.02 \\
\hline
\end{tabular}

precision, two-channel photometer that simultaneously measures the Strömgren $b$ and $y$ passbands using two EMI 9924QB photomultiplier tubes. This makes T12 ideal for achieving high photometric precision on relatively bright stars. The TSU APTs and their precision photometers, observing strategy, data reduction techniques, and photometric precision are described in detail by Henry (1999).

The T12 telescope acquired 368 nightly observations of HD 99492 during the 2004, 2009, 2010, 2013, and 2014 observing seasons. These data are plotted against Heliocentric Julian Date in the top panel of Figure 3. The observations are insufficient to detect the long-term activity cycle described in Section 2.3. Therefore, we removed very small season-toseason variability in HD 99492 and/or its comparison stars by normalizing the final four observing seasons so their means match the first season, indicated by the horizonal dotted line in the top panel. This removal of seasonal variability allows a more sensitive search for variability that might be due to rotational modulation of star spots (e.g., Henry et al. 2013). Marcy et al. (2005) estimated the rotation period of HD 99492 to be around 45 days from the $\mathrm{Ca}$ II $\mathrm{H}$ and $\mathrm{K}$ emission strength. Our nightly observations scatter about the mean with a standard deviation of $0.00484 \mathrm{mag}$, somewhat more than the typical measurement precision. However, Fourier analyses of the complete normalized data set and the individual observing seasons did not reveal any significant periodicities between 1 and 100 days that might correspond to the star's rotation period.

We further examined publicly available photometric data from the Hipparcos satellite to search for evidence of periodicity in the light curve of HD 99492 (Perryman et al. 1997; van Leeuwen 2007a). The data were extracted from the NASA Exoplanet Archive (Akeson et al. 2013), including 71 measurements spanning a period of 1062 days and with a standard deviation of $0.135 \mathrm{mag}$. Our Fourier analysis of Hipparcos did not reveal strong periodicity, with the possible exception of a minor Fourier power at $\sim 15.8$ days.

The Keplerian orbital solution in Section 3 includes an estimate of $T_{c}$, the predicted time of mid-transit should the planetary orbital inclination be suitably close to edge-on. To determine the remainder of the predicted transit parameters, we adopted the SME stellar radius from Table 1 and an estimated planetary radius of $R_{p}=0.52 R_{J}$ using the mass-radius relationship described by Kane \& Gelino (2012). Taking into account the orbital eccentricity from the Keplerian orbit (Kane \& von Braun 2008), the transit probability is $2.8 \%$ and the predicted duration and depth for a central transit are 0.181 days and $0.54 \%$, respectively.

The APT observations are replotted in the middle panel of Figure 3. These data are phased with the orbital period and the predicted transit time shown in Table 1 . We use least-squares to
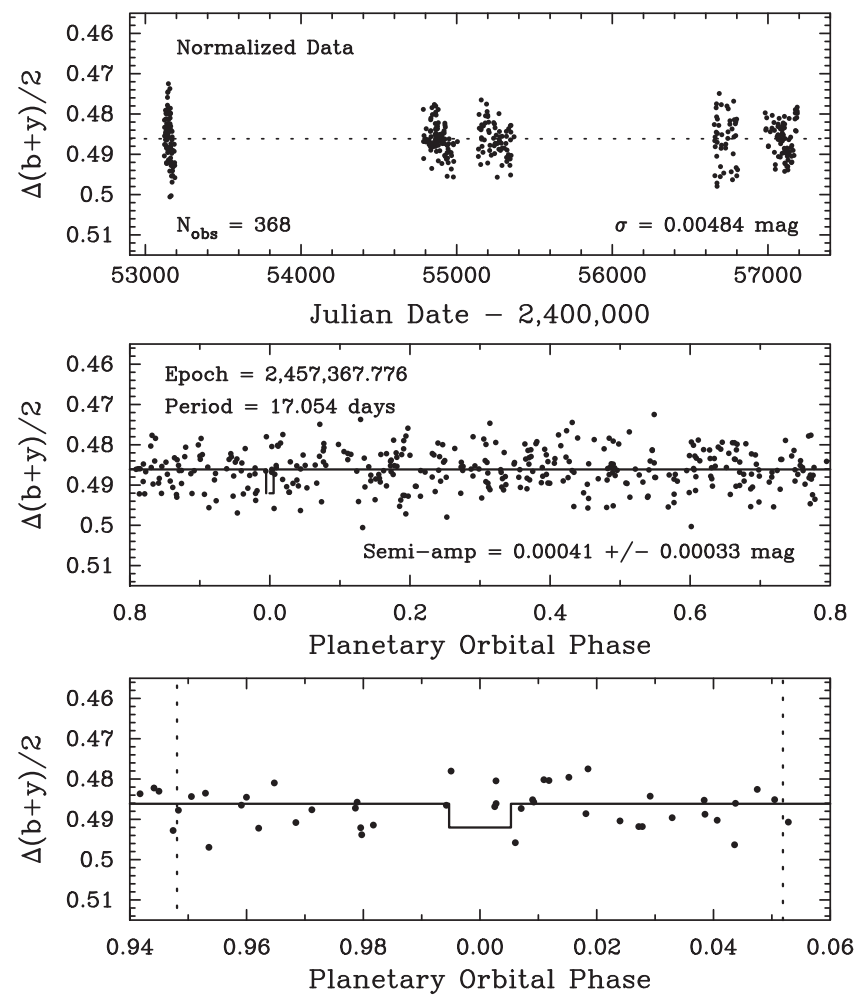

Figure 3. Top: nightly photometric observations of HD 99492 from the 2004, 2009, 2010, 2013, and 2014 observing seasons acquired with the T12 $0.8 \mathrm{~m}$ APT. The final four seasons have been normalized so their seasonal means match the 2004 season. Middle: the APT observations phased with an orbital period of 17.054 days. A sine fit to the phased observations yields a semiamplitude of $0.00041 \pm 0.00033 \mathrm{mag}$. This is consistent with the absence of light variability on the radial velocity period and also consistent with planetary reflex motion of the star as the cause of the RV variations. Bottom: the APT observations within \pm 0.06 phase units of the predicted transit time. The solid curve for the predicted transit shows the predicted mid-transit at phase 0.0 , the transit depth $(0.5 \%)$, and the duration $( \pm 0.005$ phase units) for a central transit of planet $b$. The vertical dashed lines represent the uncertainty in the time of transit. Our photometry shows no evidence for transits but cannot rule them out completely.

fit a sine curve to the data, also phased on the 17.054 day orbital period. This yields a formal semi-amplitude of the sine curve of just $0.00041 \pm 0.000033 \mathrm{mag}$. The relatively small amplitude confirms that the observed $\mathrm{RV}$ variations are due to the presence of a planet rather than intrinsic stellar brightness variations.

The APT observations within \pm 0.06 phase units of the predicted transit time are shown in the bottom panel of Figure 3 . The solid curve for the predicted transit signature includes the predicted mid-transit at phase 0.0 , the transit depth $(0.5 \%)$, and transit duration $( \pm 0.005$ phase units). The vertical dashed lines represent the uncertainty in our new time of transit. We find no evidence for transits, although our data do not rule them out completely. Monitoring observations were made on the night of 2016 January 31 UT, during a predicted transit, with the T12 APT and with the $0.6 \mathrm{~m}$ telescope at Swarthmore's Peter van de Kamp Observatory. The night was marginally photometric at both sites; again, no evidence for transits was seen but we are still not able to completely rule them out. 


\section{CONCLUSIONS}

The presence of stellar activity presents continuing challenges to exoplanet detection and characterization. RV exoplanet survey targets are usually chosen for their low chromospheric activity, leading to a bias against activity in the sample of bright planet-host stars. HD 99491 is an evolved star and has been known to exhibit chromospheric activity for some time (Zarro 1983; Wright et al. 2004). It is thus quite interesting to find that the companion star, HD 99492, exhibits similar behavior over long timescales. It is hoped that continued photometric monitoring will help to resolve the complete magentic cycle of the star, such as those found for HD 192263 (Dragomir et al. 2012), although the target is very difficult to observe due to the small angular separation of the binary components.

The update to the parameters for the HD 99492 system presented here refines the stellar and planetary orbital parameters for the system. The update shows that the $\sim 5000$ day RV signal is due to stellar activity rather than a planet. However, there are likely other planets of smaller mass and/or larger separation that lie beneath the current noise floor. As the exploration of exoplanetary systems forges onward to ever smaller planets, a careful examination of stellar activity is becoming more relevant than ever before.

G.W.H. acknowledges long-term support from Tennessee State University and the State of Tennessee through its Centers of Excellence program. This research has made use of the NASA Exoplanet Archive, which is operated by the California Institute of Technology, under contract with the National Aeronautics and Space Administration under the Exoplanet Exploration Program. The results reported herein benefited from collaborations and/or information exchange within NASA's Nexus for Exoplanet System Science (NExSS) research coordination network sponsored by NASA's Science Mission Directorate. The data presented herein were obtained at the W.M. Keck Observatory, which is operated as a scientific partnership among the California Institute of Technology, the University of California and the National Aeronautics and Space Administration. The Observatory was made possible by the generous financial support of the W.M. Keck Foundation. The authors wish to recognize and acknowledge the very significant cultural role and reverence that the summit of Mauna Kea has always had within the indigenous Hawaiian community. We are most fortunate to have the opportunity to conduct observations from this mountain.

\section{REFERENCES}

Akeson, R. L., Chen, X., Ciardi, D., et al. 2013, PASP, 125, 989

Butler, R. P., Wright, J. T., Marcy, G. W., et al. 2006, ApJ, 646, 505

Carolo, E., Desidera, S., Gratton, R., et al. 2014, A\&A, 567, A48

Demarque, P., Woo, J.-H., Kim, Y.-C., \& Yi, S. K. 2004, ApJS, 155, 667

Deming, D., Espenak, F., Jennings, D. E., Brault, J. W., \& Wagner, J. 1987, ApJ, 316, 771

Desort, M., Lagrange, A.-M., Galland, F., Udry, S., \& Mayor, M. 2007, A\&A, 473, 983

Dragomir, D., Kane, S. R., Henry, G. W., et al. 2012, ApJ, 754, 37

Dravins, D. 1985, in Proc. IAU Colloq. 88, Stellar Radial Velocities, ed. A. G. D. Philip, \& D. W. Latham (Schenectady, NY: L. Davis Press), 311

Hatzes, A. P. 2013, ApJ, 770, 133

Hatzes, A. P., Cochran, W. D., Endl, M., et al. 2015, A\&A, 580, 31

Hébrard, ÉM., Donati, J.-F., Delfosse, X., et al. 2014, MNRAS, 443, 2599

Henry, G. W. 1999, PASP, 111, 845

Henry, G. W., Kane, S. R., Wang, S. X., et al. 2013, ApJ, 768, 155

Hinkel, N. R., Timmes, F. X., Young, P. A., Pagano, M. D., \& Turnbull, M. C. 2014, AJ, 148, 54

Howard, A. W., Johnson, J. A., Marcy, G. W., et al. 2009, ApJ, 696, 75

Isaacson, H., \& Fischer, D. 2010, ApJ, 725, 875

Kane, S. R., \& Gelino, D. M. 2012, PASP, 124, 323

Kane, S. R., Mahadevan, S., von Braun, K., Laughlin, G., \& Ciardi, D. R. 2009, PASP, 121, 1386

Kane, S. R., \& von Braun, K. 2008, ApJ, 689, 492

Lodders, K., Plame, H., \& Gail, H.-P. 2009, LanB, 4, 44

Marcy, G. W., \& Butler, R. P. 1992, PASP, 104, 270

Marcy, G. W., Butler, R. P., Vogt, S. S., et al. 2005, ApJ, 619, 570

Meschiari, S., Laughlin, G., Vogt, S. S., et al. 2011, ApJ, 727, 117

Moutou, C., Mayor, M., Lo Curto, G., et al. 2011, A\&A, 527, A63

Noyes, R. W., Hartmann, L. W., Baliunas, S. L., Duncan, D. K., \& Vaughan, A. H. 1984, ApJ, 279, 763

Perryman, M. A. C., Lindegren, L., Kovalevsky, J., et al. 1997, A\&A, 323, L49

Petigura, E. A., \& Marcy, G. W. 2011, ApJ, 735, 41

Queloz, D., Henry, G. W., Sivan, J. P., et al. 2001, A\&A, 379, 279

Robertson, P., Endl, M., Cochran, W. D., MacQueen, P. J., \& Boss, A. P. 2013, ApJ, 774, 147

Robertson, P., Mahadevan, S., Endl, M., \& Roy, A. 2014, Sci, 345, 440

Robertson, P., Roy, A., \& Mahadevan, S. 2015, ApJL, 805, L22

Saar, S. H., \& Donahue, R. A. 1997, ApJ, 485, 319

Santos, N. C., Gomes da Silva, J., Lovis, C., \& Melo, C. 2010, A\&A, 511, A54

Takeda, G., Ford, E. B., Sills, A., et al. 2007, ApJS, 168, 297

Valenti, J. A., Butler, R. P., \& Marcy, G. W. 1995, PASP, 107, 966

Valenti, J. A., Fischer, D., Marcy, G. W., et al. 2009, ApJ, 702, 989

Valenti, J. A., \& Fischer, D. A. 2005, ApJS, 159, 141

Valenti, J. A., \& Piskunov, N. 1996, A\&AS, 118, 595

van Leeuwen, F. 2007a, Hipparcos, the New Reduction of the Raw Data (Berlin: Springer)

van Leeuwen, F. 2007b, A\&A, 474, 653

Vogt, S. S., Allen, S. L., Bigelow, B. C., et al. 1994, Proc. SPIE, 2198, 362

Wang, S. X., Wright, J. T., Cochran, W., et al. 2012, ApJ, 761, 46

Wilson, O. C. 1968, ApJ, 153, 221

Wright, J. T. 2005, PASP, 117, 657

Wright, J. T., \& Howard, A. W. 2009, ApJS, 182, 205

Wright, J. T., Marcy, G. W., Butler, R. P., et al. 2008, ApJL, 683, L63

Wright, J. T., Marcy, G. W., Butler, R. P., \& Vogt, S. S. 2004, ApJS, 152, 261

Zarro, D. M. 1983, ApJL, 267, L61 